\title{
Inhaltsverzeichnis vom Band 34
}

(Es erschienen Heft 1-3: 15. März 1987; Heft 4-5: 15. September 1987)

\section{Arachnida}

Broen, B. v. \& MoritZ, M.: Zum Vorkommen von Zodarion rubidum Simon, 1914, im Berliner Gebiet (Araneae, Zodariidae). . . . . . . . . . . . . . . . . . . . . 155

KARG, W.: Zur Kenntnis der Gattung Schwiebea Oudemans, 1916 (Acarina, Sarcoptiformes). . . . 141

KARG, W. \& EdLAND, T.: Neue Raubmilbenarten der Phytoseiidae Berlese, 1916 (Acarina, Parasitiformes) . . . . . . . . . . . . . . . . . . . . . . . . . 387

\section{Coleoptera}

BAEHR, M.: Pogonoglossus arfakensis sp.nov. from New Guinea (Coleoptera, Carabidae, Helluodinae) 363 BeutleR, D. \& Weidlich, M.: Einige Wasserkäferfunde aus dem Kreis Beeskow im Bezirk Frankfurt/ Oder (Coleoptera : Haliplidae, Dytiscidae) . . . . . . . . . . . . . . . . . . . . . . 351

DESENDER, K.: Distribution, the special case of sex-linked wing dimorphism and phenology of the life cycle in Trichotichnus laevicollis and T.nitens (Coleoptera, Carabidae). . . . . . . . . . 77

MAJER, K.: A revision of the genus Danacaeina ReITTER, 1887 (Col. Melyridae) . . . . . . . . . . . 355

SELANDER, R. B.: The type-species of Zonitis FABricrus and the synonymies of $Z$. flava FABricrus and Z. ruficollis FrIVALDSZKY (Col., Meloidae). . . . . . . . . . . . . . . . . . . . . . . . . 341

WINKLER, J. R.: Ekisius vitreus gen.n., sp.n., the first representative of apterous Cleridae in Oriental Region (Coleoptera, Cleridae) . . . . . . . . . . . . . . . . . . . . . . . . . . . . . . 169

\section{Diplopoda, Polydesmida}

Hoffman, R. L.: The status of the milliped genus Merodesmus Cook, 1897 (Polydesmida: Gomphodesmidae) . . . . . . . . . . . . . . . . . . . . . . 149

\section{Diptera}

BÄHRMANN, R.: Untersuchungen der Dipterenfauna in natur- und industrienahen Rasenbiotopen Thüringens (DDR) mittels Bodenfallen (Diptera Brachycera) . . . . . . . . . . . . . . . . 85

\section{Heteroptera}

GöLlNER-SCHEDLING, U.: Ergänzung und Korrektur zu der Revision der Gattung Odontoscelis LAPORTE DE CASTELnAU, 1832 (Heteroptera, Scutelleridae) . . . . . . . . . . . . . . . 217

\section{Homoptera}

PINTERA, A.: Taxonomic revision of the species of genus Chaitophorus KoCH in Palaearctis (Homoptera: Aphidoidea) . . . . . . . . . . . . . . . . . . . 219

\section{Hymenoptera}

Koch, F.: Die Gattung Ardis Konow, 1886 (Hym., Tenthredinidae). . . . . . . . . . . . . . 107

SAINI, M. S. \& SINGH, D.: Four new species of Loderus Konow from India with a key to the oriental species of this genus (Hymenoptera, Tenthredinidae). . . . . . . . . . . . . 367

Sngh, D. \& SAINI, M. S.: Five new species of Tenthredo LinNaEUs from Uttarkhand area (India) (Hymenoptera, Tenthredinidae) . . . . . . . . . . . . . . . . . . . . . . 397 


\section{Lepidoptera}

NiCulesCu, E. V.: Liste commentée des Lépídoptères Diurnes européens (Aparasternia) (Lepidoptera) 179

SINGH, G. P. \& GoEL, S. C.: Body chaetotaxy of three noctuid caterpillars (Lepidoptera) . . . . . 373

Steuer, H.: Beiträge zur Kenntnis der Elachistiden, Teil V (Lepidoptera, Elachistidae) . . . . . . . 197

\section{Odonata}

ZESSIN, W. \& ANSORGE, J.: Magnasupplephlebia intercalaria n.sp. - eine neue Anisozygopterenart aus dem oberen Lias von Mitteleuropa (Insecta, Odonata) . . . . . . . . . . . . . . . 383

\section{Orthopteromorpha}

ZESSIN, W.: Variabilität, Merkmalswandel und Phylogenie der Elcanidae im Jungpaläozoikum und Mesozoikum und die Phylogenie der Ensifera (Orthopteroida, Ensifera) . . . . . . . . . . . . . 1

INGRISCH, S. : Zur Orthopterenfauna Nepals (Orthoptera) . . . . . . . . . . . . . . . . . . 113

\section{Trichoptera}

Klima, F. \& Mey, W.: Anomalien in der Geschlechtsrealisierung bei Köcherfliegen (Trichoptera) 161 Buchbesprechungen . . . . . . . . . . . . 106, 112, 140,154, 160, 354, 362, 372, 382, 396, 406

\section{In Band 34 (1987) erschienene Neubeschreibungen}

Amblyseius latoventris W. KARG \& T. Edland n.sp. (Acari, Phytoseiidae) . . . . . . . . . . . . . . 393

Anthoseius picea W. KARG \& T. EdLAND n.sp. (Acari, Phytoseiidae). . . . . . . . . . . . . . . . . . . 389

Anthoseius toruli W. KARG \& T. EdLAND n.sp. (Acari, Phytoseiidae). . . . . . . . . . . . . . . 390

Butania metallica S. INGRISCH n.sp. (Orthoptera, Eumastacoidea). . . . . . . . . . . . . . . . . 131

Chaitophorus diversisetosus austriacus A. PINTERA ssp.n. (Homoptera, Chaitophoridae). . . . . . . 234

Chaitophorus hillerislambersi A. PINTERA sp.n. (Homoptera, Chaitophoridae). . . . . . . . . . . . 243

Chaitophorus inouyei A. PinterA sp.n. (Homoptera, Chaitophoridae). . . . . . . . . . . . . . 256

Chaitophorus melanosiphon A. PINTERA sp.n. (Homoptera, Chaitophoridae). . . . . . . . . . . . 269

Chaitophorus miyazakii A. PinterA sp.n. (Homoptera, Chaitophoridae). . . . . . . . . . . . . 272

Chaitophorus nigricantis A. Pentera sp.n. (Homoptera, Chaitophoridae) . . . . . . . . . . . . . . 279

Chaitophorus nigricantis mongolicus A. PINTERA ssp.n. (Homoptera, Chaitophoridae). . . . . . . . 281

Chaitophorus remaudierei A. Pintera sp.n. (Homoptera, Chaitophoridae). . . . . . . . . . . . 303

Chaitophorus salijaponicus stroyani A. PINTERA ssp.n. (Homoptera, Chaitophoridae) . . . . . . . . 314

Chaitophorus salijaponicus szelegiewiczi A. PINTERA ssp.n. (Homoptera, Chaitophoridae). . . . . . 317

Chaitophorus similis A. PNTERA sp.n.(Homoptera, Chaitophoridae) . . . . . . . . . . . . . . . . 321

Chaitophorus tremulae sorini A. PINTERA ssp.n. (Homoptera, Chaitophoridae). . . . . . . . . . . 326

Chaitophorus vitellinae danubicus A. PINTERA ssp.n. (Homoptera, Chaitophoridae). . . . . . . . . 334

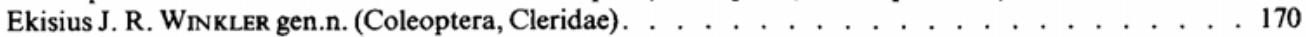

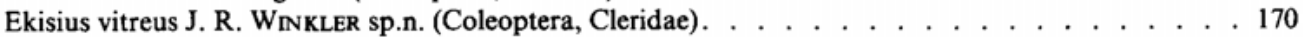

Euscyrtus intermedius S. INGRISCH n.sp. (Orthoptera, Grylloidea). . . . . . . . . . . . . . 126

Gryllopsis nepalicus S. INGRISCH n.sp. (Orthoptera, Grylloidea). . . . . . . . . . . . . . . . 125

Holochlora gaida S. INGRISCH n.sp. (Orthoptera, Tettigonioidea). . . . . . . . . . . . . . . . 120

Letana brachyptera S. INGRISCH n.sp. (Orthoptera, Tettigonoioidea). . . . . . . . . . . . . . 118

Letana nigropoda S. INGRISCH sp.n. (Orthoptera, Tettigonioidea). . . . . . . . . . . . . . . . . 116

Loderus darjeelingi M. S. SAINI \& D. SINGH sp.n. (Hymenoptera, Tenthredinidae). . . . . . . . . 369

Loderus ferrugenosa M. S. SALNI \& D. SEGH sp.n. (Hymenoptera, Tenthredinidae). . . . . . . . . 367

Loderus infuscata M. S. SAINI \& D. SINGH sp.n. (Hymenoptera, Tenthredinidae) . . . . . . . . . . 368

Loderus manalii M. S. SAINI \& D. SINGH sp.n. (Hymenoptera, Tenthredinidae). . . . . . . . . . . . 369

Magnasupplephlebia intercalaria W.ZEssin \& J. ANSORGE n.sp. (Insecta, Odonata) . . . . . . . . . . . 384

Pogonoglossus arfakensis M. BAEHR sp.n. (Coleoptera, Carabidae) . . . . . . . . . . . . . . . . 363 
Schwiebea (Jacolietta) cepa W. KARG n.sp. (Acarina, Sarcoptiformes) . . . . . . . . . . . . . . 142

Seiulus sexapori W. KARG \& T. EdLAND n.sp. (Acari, Phytoseiidae). . . . . . . . . . . . . . . . . . 388

Tenthredo floweri D. SingH \& M. S. SAINI sp.n. (Hymenoptera, Tenthredinidae). . . . . . . . . . 403

Tenthredo gobindghati D. SINGH \& M. S. SAINI sp.n. (Hymenoptera, Tenthredinidae). . . . . . . . 400

Tenthredo mandali D. SINGH \& M. S. SAINI sp.n. (Hymenoptera, Tenthredinidae). . . . . . . . . . 401

Tenthredo parapeniata D. SINGH \& M. S. SAINI sp.n. (Hymenoptera, Tenthredinidae). . . . . . . 398

Tenthredo shinoharai D. SINGH \& M. S. SAINI sp.n. (Hymenoptera, Tenthredinidae). . . . . . . . . 397

Typhlodromus eucervix W. KARg \& T. EdLAND n.sp. (Acari, Phytoseiidae). . . . . . . . . . . . 392

Xiphidonema S. INGRISCH n.gen. (Orthoptera, Tettigonoidea) . . . . . . . . . . . . . . . . 122

Xiphidonema arcuata S. INGRISCH n.sp. (Orthoptera, Tettigonoidea) . . . . . . . . . . . . . . . . 122

Xya nobile S. INGRISCH n.sp. (Orthoptera, Grylloidea) . . . . . . . . . . . . . . . . . . . . . . 127 